\title{
Aprendiendo de BBPR. El diálogo con la historia y con el contexto en las obras construidas en Palermo
}

\author{
Learning from BBPR. Dialogue with history and built context \\ in Palermo
}

$\underline{\text { G.Trovato }}^{(*)}$

RESUMEN

El artículo propone un análisis de los edificios construidos por BBPR en la ciudad de Palermo entre 1960 y 1974. En estas obras, poco conocidas por su posición geográfica marginal y por tratarse del periodo final de su carrera,la relación entre el edificio y el espacio público se defiende como principal condición para la construcción de la ciudad moderna. BBPR aquí utilizan diversas estrategias: en algunos casos el recurso a la historia, entendida como catálogo de elementos tipificados, prevalece al diálogo con el contexto específico. En la última obra, el Palazzo Amoroso, predomina el proceso inverso: se renuncia al protagonismo formal a favor de la integración en el contexto a través de la articulación volumétrica y a un uso sapiente de los materiales. Se presenta aquí el resultado de la investigación realizada en los archivos del Ingeniero Amoroso, que introdujo al grupo milanés en Palermo y les encargó el edificio homónimo.

$970-2$

Palabras clave: Arquitectura, tradición, innovación, contexto, revestimiento.
SUMMARY

The article proposes an analysis of a series of buildings by BBPR in the city of Palermo between 1960 and 1974. In these works, littleknown due to their peripheral position and because they belong to the final period of their career, the relationship between the building and the public space becomes the main condition for the construction of the modern city. BBPR here uses various strategies: in some cases they work with history, understood as a catalogue of categorized items, prevails over the dialogue with the specific context. In the last work, Palazzo Amoroso, the opposite is the case: the main quality is its discretion and its ability to interact with the environment and its complex variables, through the volumetric articulation and the intentional use of materials. We present here the research in the Engineer Amoroso's archives He introduced the Milanese group in Palermo commissioning the homonymous building.

Keywords: Architecture, tradition, innovation, context, covering
Informes de la Construcción Vol. 63, 522, 35-42, abril-junio 2011 ISSN: 0020-0883 elSSN: 1988-3234 doi: 10.3989/ic. 10.041 


\section{INTRODUCCIÓN. LA HISTORIA COMO HERRAMIENTA EN EL PROYECTO MODERNO}

Cuando el papel del arquitecto se cuestiona, quedando las competencias del proyecto edificatorio y urbanístico relegadas a ámbitos profesionales poco claros, resulta imprescindible reivindicar aquellas acciones que han dejado un sello de calidad indudable en nuestra cultura y en nuestras ciudades. "Amad a la arquitectura", decía Gió Ponti (1). El mensaje resulta fácil de transmitir en el caso de edificios históricos, entendiendo como tales a aquellos construidos en épocas clásicas o en todo caso "lejanas". Más compleja, sin embargo, es la percepción de la calidad en obras realizadas en épocas recientes, sobre todo, si son sumergidas en tejidos especulativos, surgidos de intereses económicos particulares y llevados a cabo sin un control urbanístico adecuado.

El valor histórico parece legitimar las intervenciones del hombre, a veces apreciadas a pesar de falta de calidad arquitectónica específica. Lo mismo ocurre con la utilización de elementos derivados del lenguaje arquitectónico de la antigüedad que, una vez normalizados, como dice Benévolo, pueden ser aplicados al proyecto "moderno", que resulta de esta forma más cercano a una percepción no especializada. Lo comprendieron Brunelleschi y Alberti cuando codificaron el lenguaje renacentista, reinterpretando los órdenes y los tipos derivados del pasado. Lo entendió el eclecticismo cuando, en un momento de cambios sociológicos y tecnológicos, revistió a los edificios de los estilos del pasado. La reivindicación de la historia como elemento activo en el proyecto moderno se delinea en los años que suceden a la segunda guerra mundial, como reacción a la tabula rasa del Movimiento Moderno, y se prolonga durante toda la segunda mitad del siglo XX. Los excesos del posmodernismo historicista acabarán por deslegitimar en la década de 1980 el uso cínico y banalizante de la cita del pasado, mostrando los límites de esta interpretación. En Italia la historia mantiene un peso ineludible. Eso supone una continua operación de análisis y catalogación de la arquitectura histórica, una cultura del proyecto de restauración rica de reflexión teórica pero, al mismo tiempo, el riesgo de limitar las investigaciones relativas a una posible innovación en técnicas y lenguajes.

Entre 1960 y 1974 el grupo milanés BBPR, constituido en aquel momento por Ludovico Barbiano di Belgiojoso, Enrico Peressuti y Ernesto Nathan Rogers (2), realiza cuatro obras en Palermo en las que el recurso a la historia aparece bajo distintas estrategias $(3,4)$. La primera es la tienda de óptica Randazzo, publicada en Domus en aquellos años, y hoy desaparecida (5). A ello sigue la construcción de tres edificios: entre 1962 y 1965, la sede de la Banca Comercial (hoy sucursal de Intesa Sanpaolo), situada en el ensanche de principios del siglo XX; entre 1962 y 1966, la sede de las oficinas y viviendas del Banco de Sicilia y, entre 1971 y 1974, el edificio de viviendas y locales comerciales de la Amoroso Construcciones, este último situado en los bordes del casco antiguo. Los arquitectos ya gozan en ese período de fama internacional: el proyecto icónico de la Torre Velasca, construida en Milán en 1958, ha demostrado al mundo entero la sorprendente posibilidad de aplicar el esquema de torre medieval a un rascacielos moderno, en la búsqueda de una integración con el contexto; Rogers por otro lado reviste un papel clave en el debate teórico a través de los CIAM primero y de la dirección de las Revistas "Domus. La casa dell'uomo" entre 1946 y 1947 y "Casabella. Continuità" después, entre 1953 y 1965. Junto con Sert y Tyrwhitt firma "The Hearth of de City" (6), el manifiesto con el que los CIAM propugnan el retorno a las ciudades como lugares de encuentro y agregación. Para Rogers la tradición constituye un "elemento" del proyecto moderno, es decir un "principio" o "fundamento de la teoría que determina la disciplina"(7). El concepto de "continuidad", dice Rogers, "implica el de mutación en el orden de una tradición". El discurso de Rogers viene de esta forma a representar las contradicciones del debate teórico internacional durante toda la posmodernidad (8). El papel de la historia se cuestiona como fuente de innovación y continuidad entre la tradición y un movimiento moderno que, como también Colin Rowe evidenciaba, nunca rompió con la historia, a pesar de su mensaje.

En Sicilia los arquitectos se encuentran interviniendo en tejidos consolidados, donde el diálogo con la historia y con el contexto puede manifestarse bajo distintas estrategias.

- Por un lado, la arquitectura es una disciplina con historia propia, que ofrece un repertorio de formas y elementos a interpretar a través del lenguaje moderno y del uso de nuevos materiales.

Los límites de esta interpretación pueden ser varios: en primer lugar, existe el riesgo de caer en formas y resultados previsibles por estar derivados de un tipo preestablecido; en segundo lugar, la dimensión técnica y funcional del edificio pueden quedar subordinadas a una forma de la que el proyecto deriva; por último, la continuidad con el lenguaje arquitectónico clásico no asegura la continuidad con el contexto, que a menudo resulta indiferente.

- Por otro lado, historia y tradición representan instrumentos para una lectura activa de 
los elementos formales, que puedan estar presentes en el lugar en el que se interviene. Éste deberá ser estudiado y analizado como principal fuente de inspiración, detectando la morfología de los tejidos y los esquemas agregativos presentes. Los límites de la interpretación están aquí mas abiertos a posibles interacciones con situaciones específicas, independientes de las clasificaciones tipológicas referenciales. El diálogo con el contexto prevalece al diálogo con la historia, entendida como catálogo.

\section{BBPR EN EL CONTEXTO PALERMITANO}

La aventura palermitana de BBPR empieza de la mano del ingeniero y empresario Franco Amoroso, quien, junto a su hermano, les introduce en la capital siciliana: el Giornale di Sicilia, principal periódico de la región, busca proyectista para una nueva sede que aloje las nuevas oficinas, con capacidad de dar cabida a maquinarias más modernas y viviendas para los empleados. Los hermanos proponen al estudio milanés del que conocen y aprecian la obra arquitectónica, práctica y conceptual.

La Palermo en la que están Ilamados a trabajar es una ciudad compleja, pues una fuerte irrupción de la mafia en la gestión del consistorio provoca una burbuja inmobiliaria sin precedentes. El fenómeno supone en pocos años una especulación edificatoria salvaje, con ocupación masiva del territorio municipal desde la línea de costa hasta el arco de montañas que lo delimita. Una edificación abusiva, construida sin proyecto de arquitectura previo, ahoga las villas aristocráticas y sustituye a los campos de cultivo, naranjales y limoneros prevalentemente, que desde la época árabe caracterizaban el paisaje. La ciudad asimila la nueva expansión como una capa más de sus múltiples estratos. Fragmentos romanos, recintos árabes y normandos, restos bizantinos, villas manieristas y barrocas, palacetes y pabellones decimonónicos quedan dislocados en un territorio irremediablemente comprometido. Una nueva ciudad se perfila: la burguesía se traslada a los nuevos barrios, construidos sin atención a la normativa y a la calidad del espacio público, abandonando el casco antiguo. Éste último se define como ámbito marginal en el que las sedes institucionales (Ejercito y Fuerzas Armadas, Universidad e Iglesia) conviven con el tejido popular de los antiguos mercados.

La calidad de la arquitectura y del espacio urbano, impulsada por la aristocracia local y por las instituciones hasta ese momento, se convierte en una prerrogativa de unos pocos intelectuales y promotores que intentan impulsar la cultura del proyecto como el Movimiento Moderno lo entendía. A las

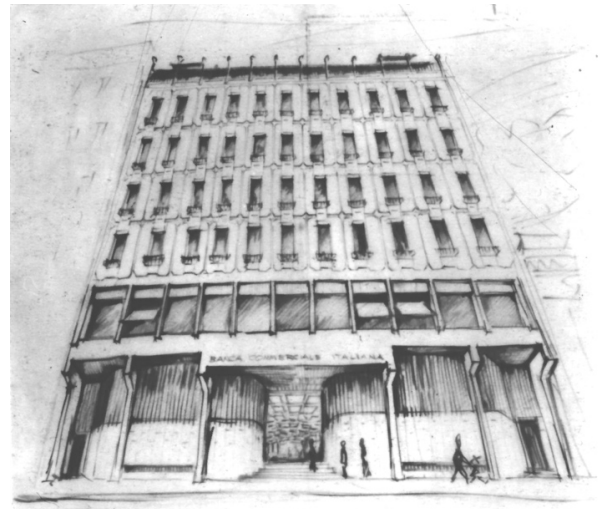

1. Croquis de la fachada de la sede de la Banca Comercial (hoy Intesa San Paolo) situada en el ensanche de principios del siglo $\mathrm{XX}$. energías de arquitectos locales como Gianni Pirrone y Leonello Foderá se une el papel de los arquitectos viajeros, como Urbani define a los profesionales de prestigio que bajaban a la isla desde la península (9). Entre ellos cabe destacar a Giuseppe Samonà, de origen palermitano, que en 1961 construye la sede della SGES, hoy Enel, en el nuevo ensanche.

Los hermanos Amoroso colaboran con algunos de ellos promoviendo una arquitectura de calidad en el ensanche y en el casco antiguo. Mientras los arquitectos milaneses empiezan a trabajar en el proyecto de la óptica, los constructores gestionan un complejo proceso de adquisición de terrenos sobre los que se elevará la última obra del periodo palermitano.

En este arco de tiempo el grupo BBPR entra en contacto con la ciudad, con los estilos superpuestos en épocas diferentes, los materiales históricamente más utilizados, las articulaciones volumétricas y los colores de las fachadas. Cada proyecto estará precedido de un análisis atento del contexto y de las variables que lo configuran. Cada zona en la que intervienen tiene una connotación específica pues Palermo es una ciudad estratificada y con rostros distintos.

\section{EL DIÁLOGO CON LA HISTORIA Y CON EL CONTEXTO}

A partir de 1962 los BBPR empiezan a trabajar en dos proyectos al mismo tiempo. Se trata de dos programas funcionales mixtos con oficinas y viviendas, como ocurría en el proyecto de la torre milanesa. La Sede de la Banca Commerciale surge en el ensanche de principios del siglo XX, en alineación con una edificación relativamente anodina que lleva a los arquitectos a refugiarse en un repertorio tradicional, sin buscar conexiones específicas con el entramado urbano. El edificio se plantea como la elegante representación arquitectónica y urbana de una Institución. El esquema de fachada es por lo tanto clásico, articulado en base, cuerpo y coronación, cada uno tratado con materiales diferentes (Figura 1). El eje de simetría queda enfatizado 
2. Sede de la Banca Comercial. Foto de la escalera que conecta la planta baja con el sótano. Las decoraciones floreales son del artista siciliano Renato Guttuso.

3. Croquis de la solución no realizada para la sede de las oficinas y viviendas de los empleados del proyecto para la sede de las oficinas y viviendas de los empleados del Giornale di Sicilia. Giornale di Sicilia.

4. Croquis de la solución final del
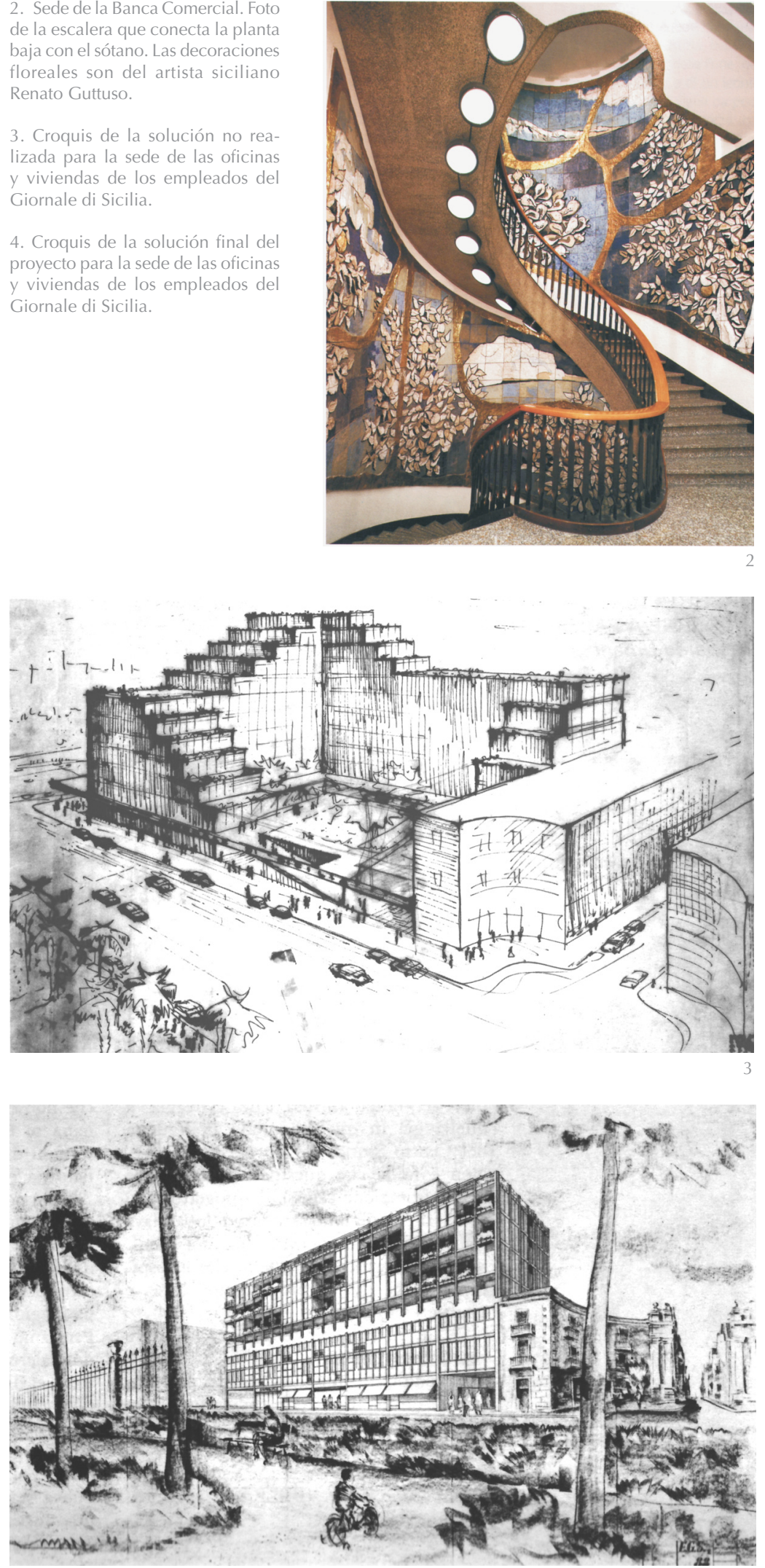

por el tratamiento del portal y del zócalo de base. El cuerpo del edificio sobresale y se reviste con una trama de paneles de mármol que revelan su espesor en la separación entre elementos. El eje central se acentúa en planta a través de la gran vidriera realizada en técnica mixta por el pintor Renato Guttuso a medida para el salón central del edificio. Lo atestigua la carta con la Belgiojoso invita al artista siciliano a visitar el estudio para trabajar juntos en la maqueta, donde la vidriera se sitúa. (10) Se retoma así una relación empezada con ocasión de la Trienal de Milán de 1934 y basada en pasiones y preocupaciones comunes, como son el interés por las tradiciones locales, que inspiran a Guttuso las escena de pesca reflejada en la vidriera, y el gusto por el neo-liberty que aparece en las decoraciones de la escalera helicoidal que conecta la planta baja con el sótano del edificio (Figura 2).

El segundo proyecto es la Sede del Giornale di Sicilia. Los arquitectos elaboran unas interesantes propuestas previas que finalmente derivarán en un proyecto menos original en el que parecen citarse a sí mismos. El programa funcional es complejo: se trata de proyectar unas oficinas capaces de alojar como hemos visto nuevas maquinarias de grandes dimensiones destinadas a una producción editorial más moderna, además de proporcionar alojamiento a las familias de los empleados. Por otro lado el solar está situado en un lugar singular en el borde del casco antiguo, en proximidad del mar, y en frente del Jardín Botánico. Esta posición quizás haya inspirado la primera propuesta, sucesivamente abandonada, de macroestructra escalonada alrededor de un patio central, elevado sobre una base continua. El proyecto tenía interés por diversos motivos: las terrazas se organizaban como jardines - miradores, con zonas verdes y amplias vistas al paisaje; el patio por otro lado quedaba comunicado a través de una rampa continua con el espacio público, configurándose así el interior del edificio como una ampliación del mismo. El punto no resuelto, fuera, quizás, la conexión con el edificio histórico colindante, la Porta Reale, al que éste parecía dar la espalda (Figura 3). La solución final, finalmente bebe del repertorio formal utilizado por el Estudio en la elaboración de programas funcionales híbridos parecidos como el de la propia Torre Velasca o el edificio de oficinas y viviendas de la Plaza del Estatuto construido en Turín en el mismo año. Se reconoce por ejemplo la dialéctica estructura - revestimiento, investigada por Rogers en la obra de August Perret al que dedica en 1955 un ensayo (Figura 4). En el libro queda reflejado que la estructura es un elemento expresivo que confiere ritmo y orden al edificio (11).

\section{PALAZZO AMOROSO: REDEFINIR EL CONTEXTO CON UN PROYECTO MODERNO}

Diverso es el enfoque en el proyecto del Palazzo Amoroso, último proyecto de la etapa palermitana. Cuando los constructores empiezan la cimentación del edificio, en 1971, Rogers ha muerto (Rogers muere en 1969, Peressutti en 1976 y Belgiojoso en 2004). Sin embargo, 
ha participado en la redacción del proyecto, constituyendo en todo momento el referente teórico con el que se debaten en conjunto las ideas proyectuales. Peressuti será el interlocutor constante y el viajero más asiduo en Palermo.

En este caso el proceso es inverso a los analizados precedentemente: los arquitectos, libres por elección de los promotores en sus planteamientos formales y estructurales, parten de soluciones tipológicas más previsibles, para evolucionar hacia una depuración progresiva del lenguaje. A la contraposición estructurarevestimiento optan aquí por la continuidad de la piel y, a través de su tratamiento superficial, establecen las claves para una relación no mimética con el contexto.

El proyecto surge en uno de los lugares más bellos del casco antiguo de Palermo, en la convergencia entre el eje estructurante de la ciudad antigua y la muralla, en proximidad de la Puerta Felice y del mar. Sustituye a las ruinas de dos palacios históricos destruidos por los bombardeos aliados en la Segunda Guerra Mundial: el Palacio de Spucches, en el vicolo de la Zecca (paralelo al Cassaro) y el Palacio Massa en la Piazzetta Santo Spirito. Del primero quedan, en el momento de la intervención, fragmentos de la fachada principal con el portal barroco y el correspondiente balcón de la planta noble (Figura 5).

La primera operación por tanto consiste en decidir la articulación volumétrica, que tendrá claramente la voluntad de manejar el vocabulario histórico del lugar a través del uso de las formas, los materiales y los colores presentes en la ciudad. En las primeras anotaciones dicen: "El esquema de proyecto aquí presentado quiere ser: 1 una indicación de los límites volumétricos impuestos por la normativa; 2 una indicación del espíritu en el que se entiende realizar este edificio en el ambiente histórico- edilicio existente. Esta ambientación se llevará a cabo a través del uso del color, de los materiales y de las formas. El color del revoco (...) será él típico de los edificios colindantes, es decir un ocre-rosado que es el color de la piedra de Aspra con la que está construido el portal barroco en el lado derecho del edificio en construcción (el portal será conservado in loco después de un proceso de restauración previo). La piedra de Aspra será utilizada

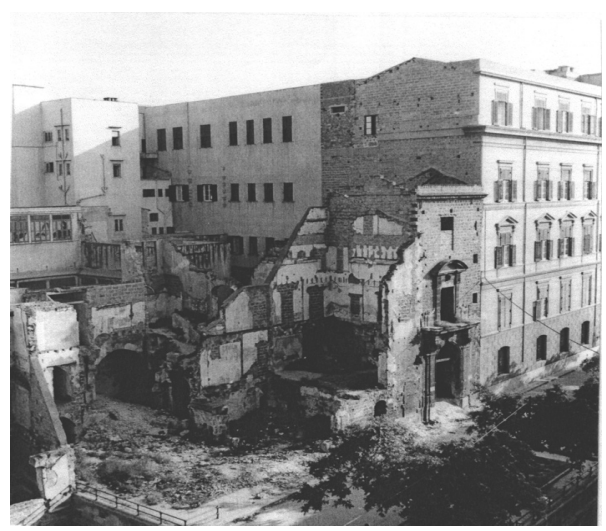

5. Las ruinas del Palacio De Spucches antes de la construcción del Palazzo Amoroso (archivo Amoroso).

6 y 7. Láminas con planteamientos iniciales que quieren reflejar según los arquitectos "el espíritu según e cual se pretende construir el edificio" archivo Amoroso).

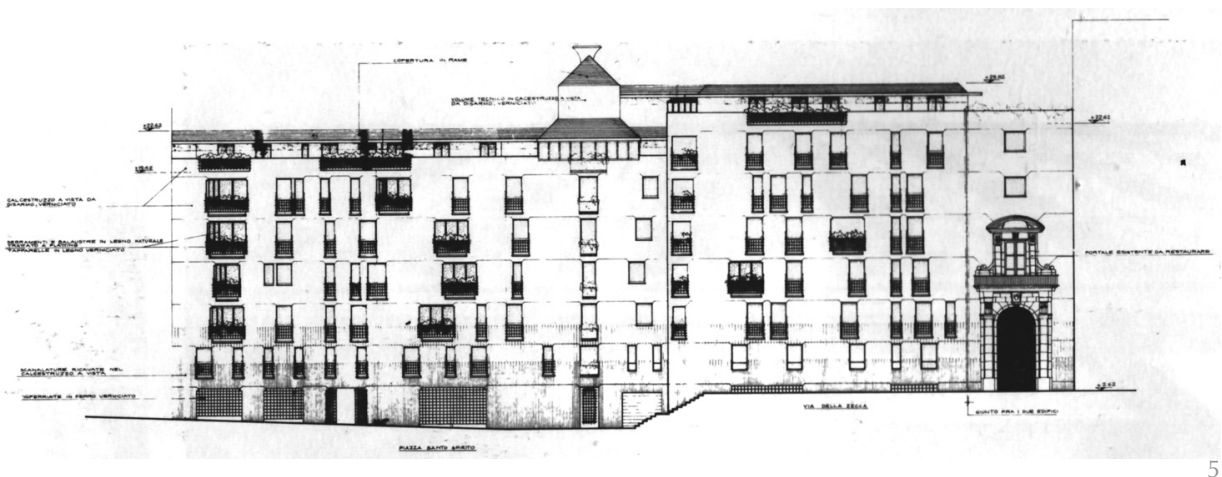

el papel de conexión y re-composición de los antiguos tejidos. Para ello BBPR optan por retomar el trazado urbano reinterpretándolo e incorporando las ruinas en el proyecto moderno. El edificio es por tanto el resultado de la articulación de dos volúmenes: uno prismático que conserva las preexistencias barrocas y otro irregular, que retoma el trazado del Palacio Massa. Cada cuerpo tiene un acceso propio.

Su forma armoniza con la Plaza y subraya el camino al colindante Palacio Butera.

La intención de capturar el "espíritu del lugar" queda reflejada en los croquis iniciales donde los arquitectos manifiestan

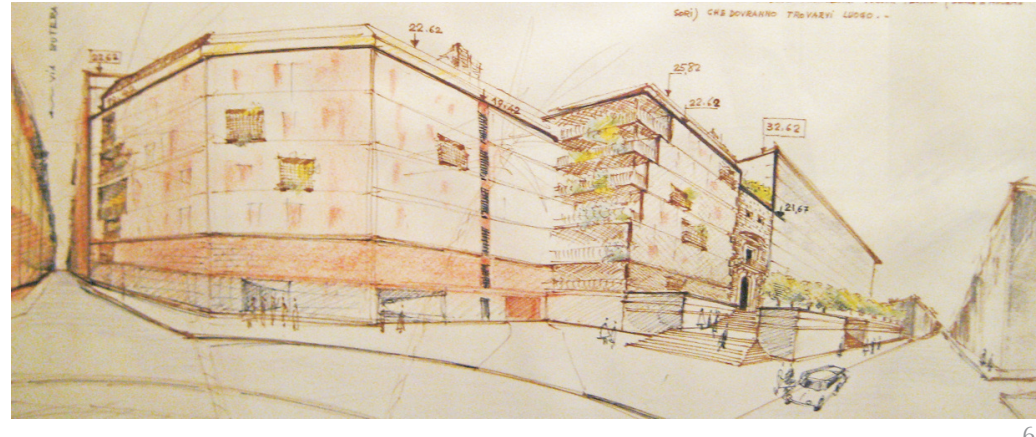

como material para el zócalo, las cornisas, etc. El hierro batido natural se empleará en los petos y en la protección de las terrazascuadradas (...)" (Figuras 6 y 7 ). 
8. Maqueta de una segunda solución, en la que los fragmentos del portal barroco se diferencian del resto de la fachada. La diferenciación desaparece en la versión realizada.

9 y 10. Planta baja y axonométrica de los duplex.
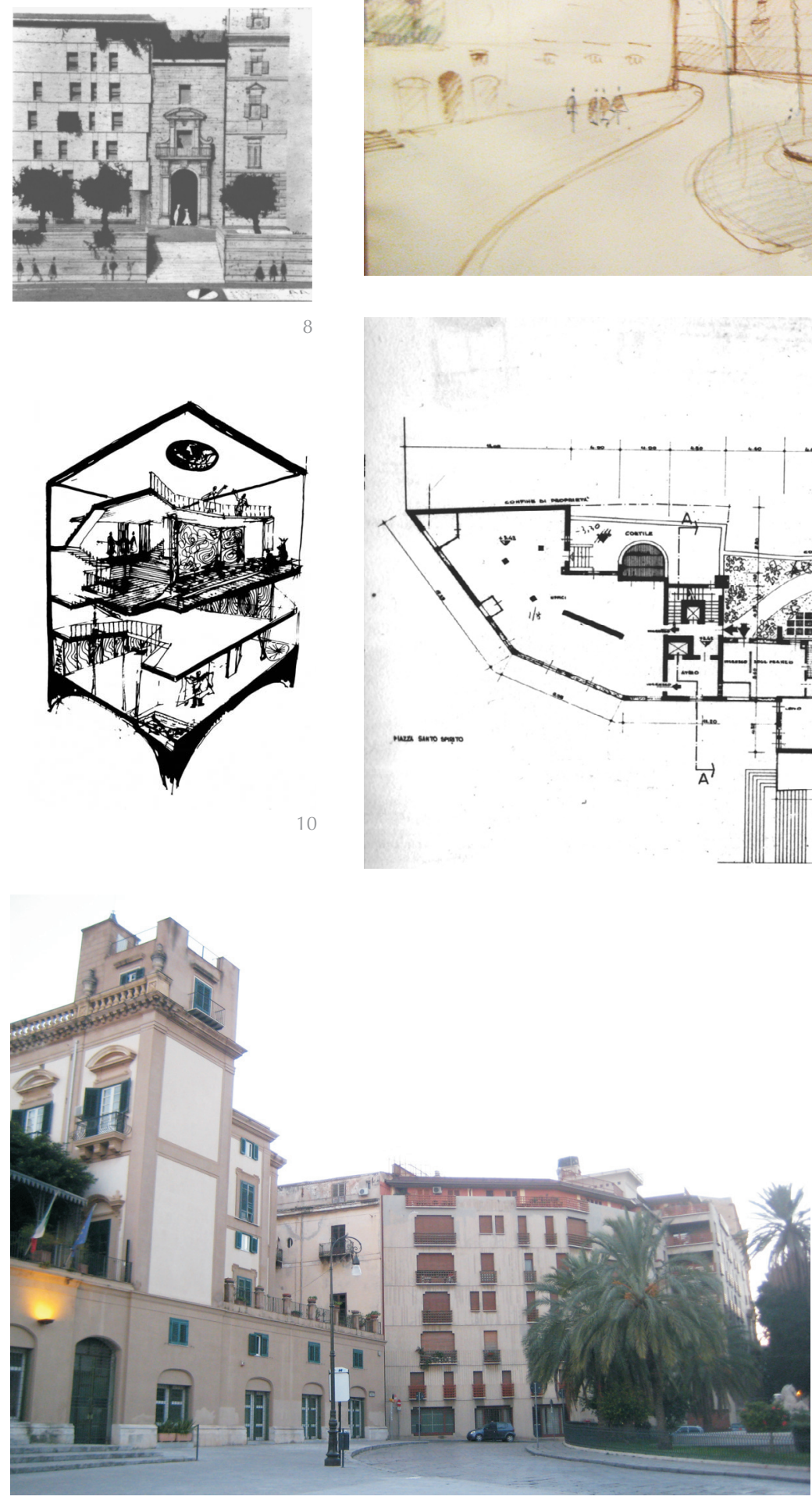

11. Vista de la Piazzetta Santo Spirito. Se aprecia la integración del edificio en el contexto histórico.
Estas intenciones iniciales se traducen por tanto en una primera propuesta clásica, donde el zócalo en piedra está diferenciado del cuerpo
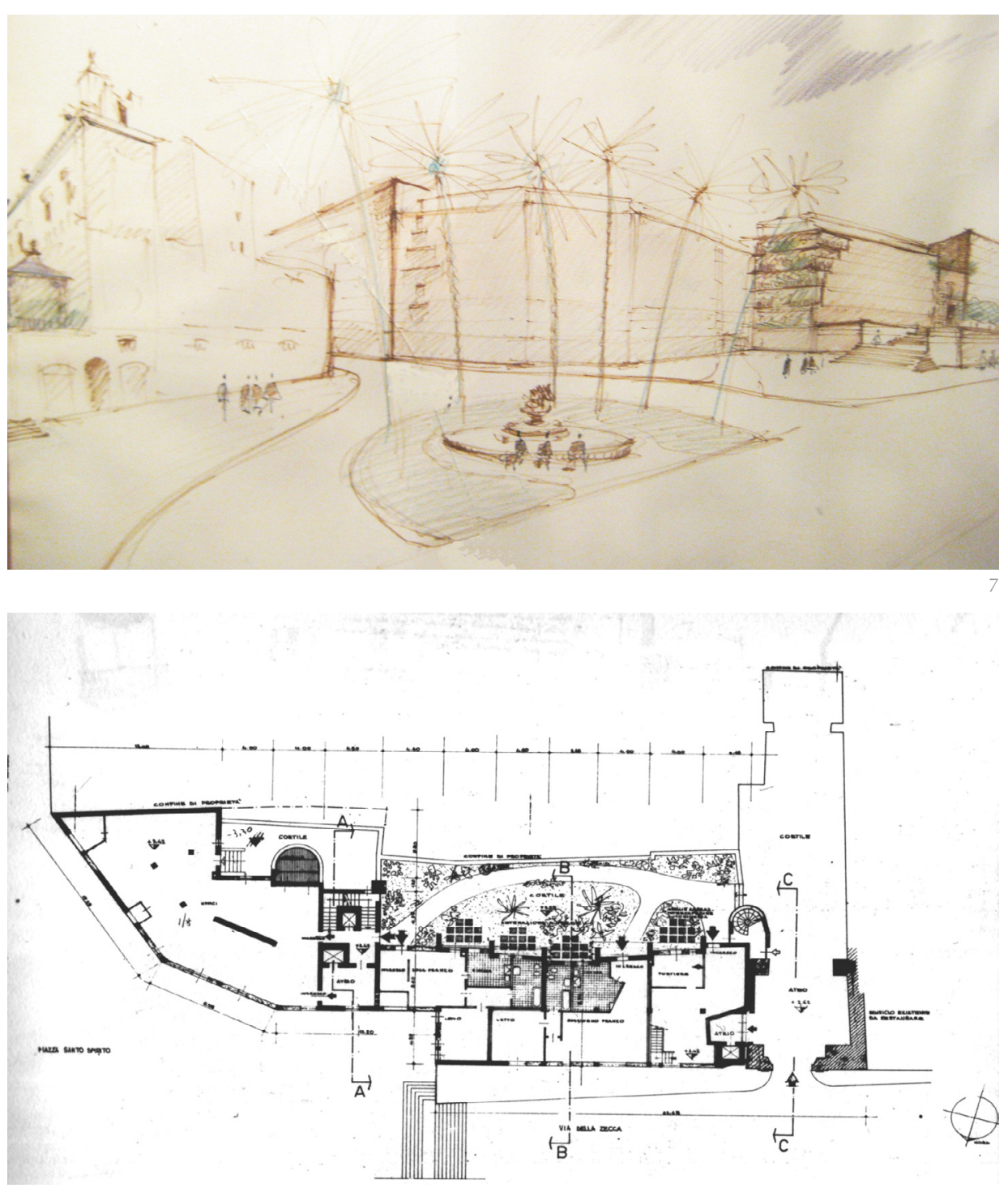

del edificio, tratado con pintura ocre, como los palacios tradicionales. Por otro lado aparece la solución de terrazas escalonadas propuesta para la sede del banco de Sicilia, que sirven aquí de charnela entre los dos volúmenes. Las ruinas están recortadas con un carácter romántico de fragmento pintoresco en la fachada, solución que desaparece en la solución definitiva (Figura 8). Ésta última se construye finalmente con paneles modulares de hormigón visto tratados sutilmente con un rayado superficial que sugiere una jerarquía en la fachada, sin que ésta se perciba de forma explícita. Zócalo, alfeizares de ventanas y cornisas se dibujan a través del tratamiento del revestimiento y del juego de sombras que éste produce. El diálogo con el contexto prevalece finalmente al diálogo con la historia, entendida como catálogo de soluciones establecidas, que queda así abstraído en juego de líneas y sugerencias abiertas a la libre interpretación (Figuras 9 y 10). El hormigón visto

se mantiene en los interiores de las viviendas en las que aparecen los pilares y las vigas con el mismo tratamiento. 
En la redefinición del espacio público intervienen los elementos de mobiliario urbano, que los arquitectos diseñan con el mismo lenguaje y con los mismos materiales, con función de filtro al acceso al edificio, (Figuras 11 y 12).

La cualidad principal de Palacio Amoroso consiste finalmente en su discreción y capacidad de interactuar con el entorno y las complejas variables que lo configuran (Figuras 13 a 15). En este sentido podemos hablar probablemente de uno de los ejemplos más acertados de cómo construir en lo construido, en una integración sobria y elegante al mismo tiempo.

\section{AGRADECIMIENTOS}

Por el portal barroco del Palazzo Amoroso, se accede, además, al estudio de la Amoroso Construcciones, donde se guardan los archivos de la empresa y del proyecto realizado con BBPR. Quiero expresar mi agradecimiento al Ingeniero Amoroso, promotor de la obra homónima, por haberme recibido en su casa y en su estudio, con sede como decíamos, en el mismo edificio, en enero de 2010. De la entrevista, realizada en esa ocasión, y de la visión del material guardado en los archivos, a los que se ha tenido acceso, se han extraído algunos de los datos contenidos en el artículo. También agradezco a Pina di Francesca y a Gabriele Pucci, por su ayuda en la investigación. En el Palazzo Amoroso también se encuentra la Fundación Rosario La Duca, historiador siciliano, que vivió en el edificio hasta su muerte, en el año 2008.

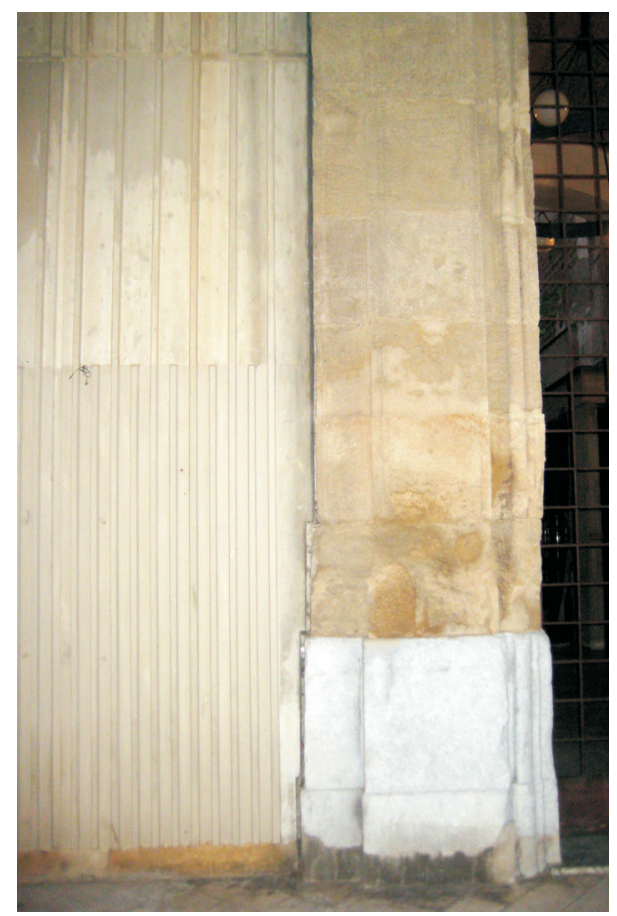

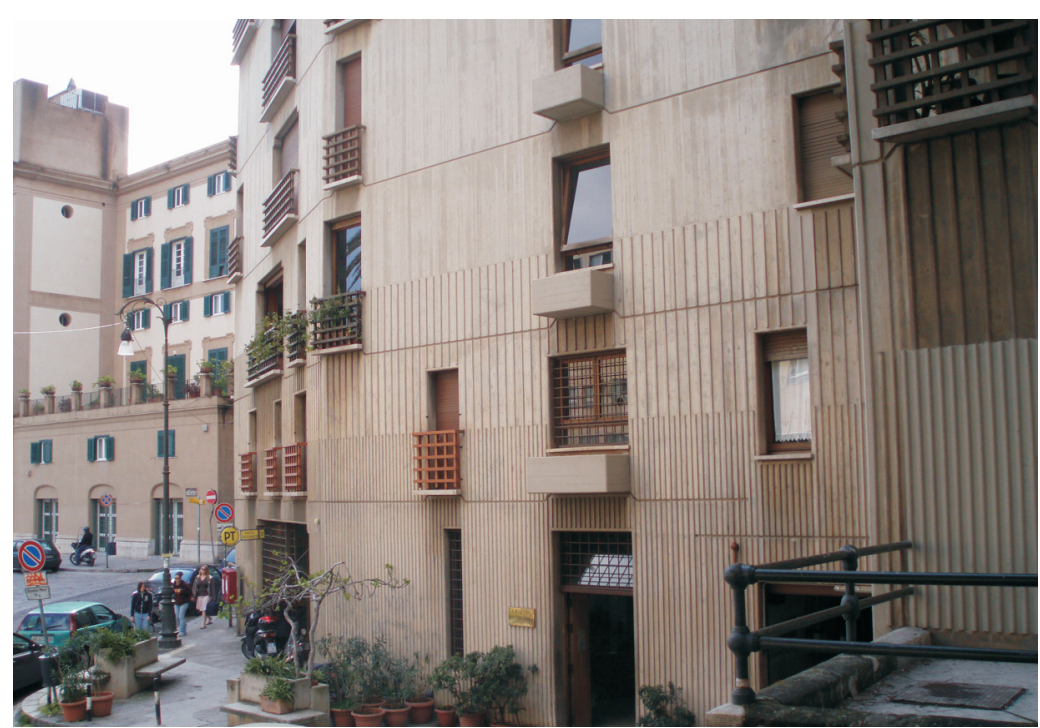

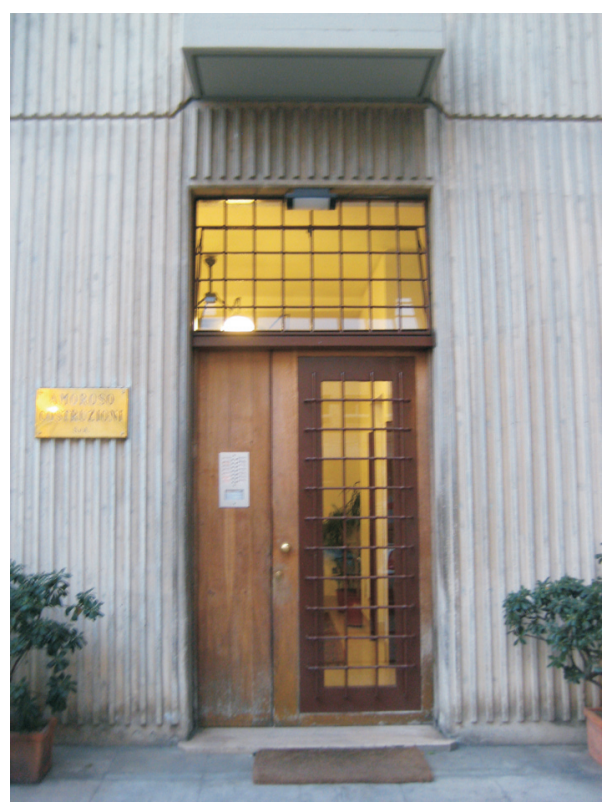

12. Vista del edificio en la que se evidencia el tratamiento de la superficie, constituida por paneles en hormigón visto. La jerarquía de la fachada se establece a través de un juego de líneas que apunta a la diferenciación clásica de la fachada en base, cuerpo y coronación.

13. Puerta de acceso por la Piazzetta Santo Spirito.

14. El encuentro entre los paneles en hormigón visto y el Portal barroco del destruido Palacio De Spucches.

15. Detalle de la fachada en el Vicolo della Zecca.

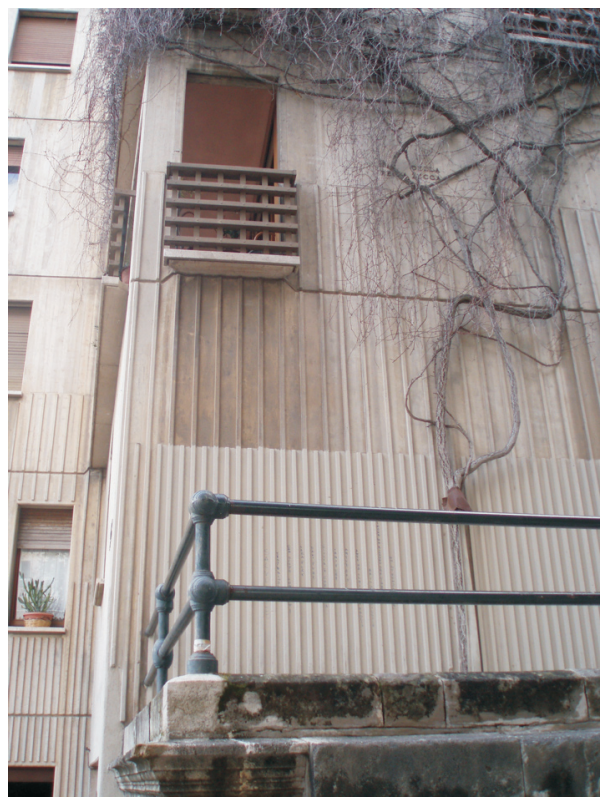




\section{BIBLIOGRAFÍA}

(1) Ponti, G.: "Amate I'architettura". Rizzoli Libri Illustrati, Milán, 2008 (primera edición 1957).

(2) Maffioletti, S.: BBPR. P. 236-238, Zanichelli. Bolonia, 1994.

(3) Sciascia, A.: "Architettura contemporanea a Palermo". p. 74-82. L’Epos, Palermo, 1998.

(4) Iannello, M.; Scolaro, G.: Palermo Guida all'architettura del '900. Salvare Palermo, Palermo, 2009.

(5) BBPR: "Un negozio di foto-ottica a Palermo". Domus no 383, Octubre (1961).

(6) Tyrwhitt, J.; Sert, J.L.; Rogers, E.N. (eds.). The Hearth of the City. Towards the humanisation of urban life, CIAM 8, Londres, 1952.

(7) De Seta, C.; Rogers, E.N.: Gli elementi del fenomeno architettonico. p. 25 y 69, Christian Marinotti Edizioni srl., Milán, 2006.

(8) López Reus, E.: Ernesto Rogers y la arquitectura de la Continuidad. Ediciones Universidad de Navarra, S.A. Pamplona, 2002.

(9) Urbani, L.: Verso un disegno per Palermo. p. 23-28 Le Architetture moderne a Palermo. M.ED.IN.A. Palermo 1986. Catalogo de la Exposición.

(10) Giordano, M.: "Guttuso e BBPR a Palermo", Kalòs. Arte in Sicilia no 1 (2009), pp. 15-17.

(11) Rogers, E.N.: Auguste Perret. II Balcone, Milán, 1955. 